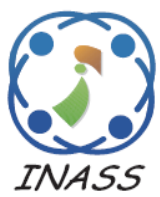

\title{
On the Performance Balancing for Uplink NOMA Systems
}

\author{
Nam-Soo Kim ${ }^{1 *}$ \\ ${ }^{I}$ Deptartment of Electronic Engineering, Cheongju University, Republic of Korea \\ * Corresponding author's Email: nskim@cju.ac.kr
}

\begin{abstract}
Outage probability and capacity are the representative performance measures for the quality of service (QoS) in mobile cellular systems. Recently, power back-off scheme is proposed in uplink non-orthogonal multiple access (NOMA) systems. The power back-off scheme improves the performance of a near user, however, decreases that of a far user. In comparison, the scheme indicates the error floors with an outage probability of $2.4 \times 10^{-1}$ and $9.1 \times 10^{-2}$ with power back-off $5 \mathrm{~dB}$ and $10 \mathrm{~dB}$, respectively under the specified condition. To address these drawbacks, we propose an equal average signal-to-interference plus noise ratio (SINR) scheme that derives the same average SINR from active users at the base station (BS) in uplink non-orthogonal multiple access (NOMA) systems. Numerical results show that required signal-to-noise ratio (SNR) for the outage probability of $1 \times 10^{-3}$ of the near and far users are close enough within $1 \mathrm{~dB}$, which means an outage balance between two users. And it is noticed that the outage probabilities in the proposed scheme decrease as the increase of the received SNR without error floors. Also, different from the power back-off scheme, we noticed that the capacities of the two users in the proposed scheme are coincident and increase with SNR. The outage probabilities and ergodic capacity of the near and far users are derived in closed-form expressions. The analytical results are conformed by Monte Carlo simulation.
\end{abstract}

Keywords: Ergodic capacity, NOMA, Performance balancing, Power control, Uplink NOMA.

\section{Introduction}

Recently, the data traffic of mobile cellular systems has increased rapidly. For faster processing of the increased traffic, high spectral efficiency is required in next generation cellular system. Nonorthogonal multiple access (NOMA), which has high spectral efficiency by transmitting multiple user's signal simultaneously in the same communication resource block, has been focused as a candidate for the next generation system [1-3].

Since Y. Saito et al. proposed the NOMA protocol in cellular systems first [4], most of the studies have been devoted to the performance analysis and the improvement of downlink NOMA systems [5-8]. More recently, the focus is moving toward the analysis of uplink NOMA systems; user performance of uplink NOMA [9], uplink cooperative multipoint transmission [10], cooperative relay [11], and power control in uplink NOMA system [12-14].
To maintain the required communication quality of users, irrespective of the location of users in a cell, it is necessary the transmit power from the different users must be controlled. Generally, the transmit power control of a user equipment is critical in conventional terrestrial cellular system to avoid inter user interference. If the transmit power is identical irrespective of the user location, the received signal strength from a near user is stronger than that of the far user. This causes interferences to the far user in the adjacent channel in frequency division multiple access (FDMA) system and on the same channel in code division multiple access (CDMA) system. In long term evolution (LTE) system, the transmit power of a user equipment (UE) is controlled to satisfy the same target power at a base station (BS).

Recently, N. Zhang et al. proposed a power backoff scheme of which a far user transmits lesser power compared to a near user in an uplink NOMA system [12]. This scheme consumes less power for the far user and reduces the interference to neighbour cells. The performance of the scheme can be improved by 
considering the decoding order. In 2017, Y. Gao et al. adapted dynamic decoding order according to the instantaneous channel state information (CSI) for the outage performance of a power back-off scheme [13]. The performance of the power back-off scheme in conjunction with rate splitting was published [14].

Although the power back-off scheme reveals that the outage probability of a near user can be improved, however, the outage probability of a far user is increased due to the less transmit power. And the error floors, which does not decrease with the increase of the received signal-to-noise ratio (SNR), are observed for both of the near and far users.

Motivated by these previous studies, we propose an equal average signal-to-interference plus noise ratio (SINR) scheme for uplink NOMA systems. The key benefits of the proposed scheme can be described as follows; firstly, the outage performances of the near user and far user are very close, which means the outage balance between users. Secondly, the error floors can be avoided and the outage probabilities decrease with the increase of the received SNR. Thirdly, the equivalent ergodic capacity of different users can be obtained, which is not guaranteed in the back-off power scheme.

The remainder of this paper is organized as follows. Section 2 describes the uplink NOMA system model and the received SINR at a BS in a cell. Section 3 derives the performance analysis of outage probability and ergodic capacity for the proposed scheme. The numerical examples and observations of the proposed and the conventional schemes are given in section 4. Finally, section 5 summarizes this work.

\section{Uplink NOMA system model}

We consider an uplink scenario of a NOMA system with a base station (BS) and two active user equipment (UE) in a single cell as shown in Fig. 1.

The received signal at the BS can be written by

$$
r=\sum_{i=1}^{2} \sqrt{P_{r i}} h_{i} x_{i}+n
$$

where $P_{r i}$ and $x_{i}$ are the received power and the information from the ith UE, respectively, with $\mathrm{E}\left[\left|x_{i}\right|^{2}\right]=1 . h_{i}$ denotes the channel coefficient between the BS and the ith UE, which has complex Gaussian distribution with zero mean and unit variance, $h_{i} \sim C N(0,1) . n$ is the complex Gaussian noise with zero mean and the variance of $N_{0}, n \sim$ $\mathrm{CN}\left(0, N_{0}\right)$. Without loss of generality, UE1 and UE2 are denoted as near and far user, respectively.

The received SINR for decoding $x_{1}$ at the BS can be written as

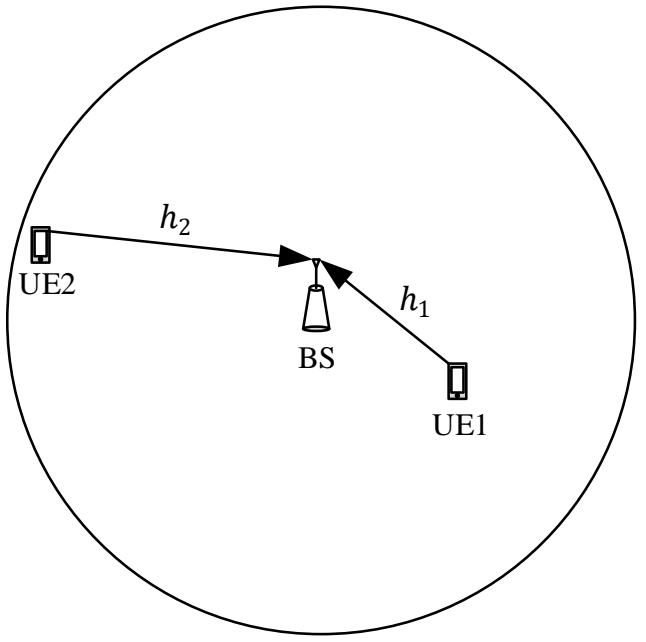

Figure. 1 Uplink NOMA system model

$$
\gamma^{x_{1}}=\frac{P_{r_{1}}\left|h_{1}\right|^{2}}{P_{r_{2}}\left|h_{2}\right|^{2}+N_{0}}=\frac{\rho_{1}\left|h_{1}\right|^{2}}{\rho_{2}\left|h_{2}\right|^{2}+1}
$$

where $\rho_{1}$ and $\rho_{2}$ are the received average SNR from UE1 and UE2, respectively, with $\rho_{1}=P_{r 1} / N_{0}$ and $\rho_{2}=P_{r 2} / N_{0}$. And the received SINR for decoding $x_{2}$ at the BS can be expressed as

$$
\gamma_{\text {no SIC }}^{x_{2}}=\frac{P_{r 2}\left|h_{2}\right|^{2}}{P_{r 1}\left|h_{1}\right|^{2}+N_{0}}=\frac{\rho_{2}\left|h_{2}\right|^{2}}{\rho_{1}\left|h_{1}\right|^{2}+1} \text {. }
$$

We assume the decoding of the information from UE1 is done prior to that from the UE2, the interfering component $x_{1}$ from UE1 can be removed by successive interference cancellation (SIC) process. After SIC, the SINR of Eq. (3) can be rewritten by

$$
\gamma^{x_{2}}=\frac{P_{r 2}\left|h_{2}\right|^{2}}{N_{0}}=\rho_{2}\left|h_{2}\right|^{2}
$$

For the decoding order, we will describe in the next section.

\section{Performance analysis}

In this section, we consider the outage probability and ergodic capacity.

\subsection{Outage probability}

When the received SINR at the BS bellows the threshold, the outage event happens. And the outage probability of UE1 can be written by

$$
P_{o_{-} U 1}=\operatorname{Pr}\left(\gamma^{x_{1}}<\Gamma\right)
$$

where $\Gamma, \Gamma=2^{R_{1}}-1$, is the threshold, and $R_{1}$ is the spectral efficiency of UE1 in [bps/Hz]. By replacing 
Eq. (2) into Eq. (5), the outage probability can be rearranged by

$$
\begin{aligned}
& P_{o_{U 1}}=\operatorname{Pr}\left(\left|h_{1}\right|^{2}<\frac{\Gamma\left(\rho_{2}\left|h_{2}\right|^{2}+1\right)}{\rho_{1}}\right) \\
= & \int_{0}^{\infty}\left(1-e^{-\frac{\Gamma\left(\rho_{2} x+1\right)}{\rho_{1}}}\right) e^{-x} d x=1-e^{-\frac{\Gamma}{\rho_{1}} \frac{1}{\frac{\Gamma \rho_{2}}{\rho_{1}}+1}}
\end{aligned}
$$

where the second equality holds from Rayleigh probability density function (pdf) for the channel coefficient. And the outage events for the UE2 happen two cases; firstly, when the decoding of $x_{1}$ fails, and secondly, when the decoding of $x_{2}$ fails though the decoding of $x_{1}$ success. The outage probability of UE2 can be given by

$$
P_{o_{-} U 2}=\operatorname{Pr}\left(\gamma^{x_{1}}<\Gamma\right)+\operatorname{Pr}\left(\gamma^{x_{1}} \geq \Gamma, \gamma^{x_{2}}<\Gamma\right)
$$

where the second probability of Eq. (7) can be rearranged from Eq. (2) and Eq. (4),

$$
\begin{aligned}
& \operatorname{Pr}\left(\gamma^{x_{1}} \geq \Gamma, \gamma^{x_{2}}<\Gamma\right) \\
& \quad=\operatorname{Pr}\left(\left|h_{1}\right|^{2} \frac{\Gamma\left(\rho_{2}\left|h_{2}\right|^{2}+1\right)}{\rho_{1}},\left|h_{2}\right|^{2}<\frac{\Gamma}{\rho_{2}}\right) .(8)
\end{aligned}
$$

For the notational convenience, denote $\left|h_{1}\right|^{2}=U$, and $\left|h_{2}\right|^{2}=V$. Then the probability of Eq. (8) can be written by

$$
\begin{aligned}
& \operatorname{Pr}\left(\gamma^{x_{1}} \geq \Gamma, \gamma^{x_{2}}<\Gamma\right) \\
= & \int_{v=0}^{\Gamma / \rho_{2}} \int_{u=\Gamma\left(\rho_{2} v+1\right) / \rho_{1}}^{\infty} f_{U, V}(u, v) d u d v
\end{aligned}
$$

where $f_{U, V}(u, v)$ is the joint pdf of $U$ and V. Since the random variables of $U$ and $V$ are independent each other, the probability of Eq. (9) can be obtained as

$$
\begin{aligned}
\operatorname{Pr}\left(\gamma^{x_{1}} \geq \Gamma, \gamma^{x_{2}}<\Gamma\right) \\
\quad=\int_{v=0}^{\frac{\Gamma}{\rho_{2}}} \int_{u=\frac{\Gamma\left(\rho_{2} v+1\right)}{\rho_{1}}}^{\infty} e^{-(u+v)} d u d v \\
=e^{-\Gamma / \rho_{1}} \frac{1}{\frac{\Gamma \rho_{2}}{\rho_{1}}+1}\left(1-e^{-\left(\frac{\Gamma^{2}}{\rho_{1}}+\frac{\Gamma}{\rho_{2}}\right)}\right)
\end{aligned}
$$

where the first probability holds from the exponential pdf for U and V, respectively. Replacing Eq. (10) into Eq. (7), we can obtain the outage probability of UE2 as

$$
P_{O_{-} U 2}=1-\frac{1}{\frac{\Gamma \rho_{2}}{\rho_{1}}+1} e^{-\left(\frac{\Gamma+1}{\rho_{1}}+\frac{1}{\rho_{2}}\right) \Gamma} .
$$

\subsubsection{Proposed equal average SINR scheme}

The dominant factor for QoS in an orthogonal multiple access (OMA) system is SNR. Different from OMA, the inherent inter-user interference in NOMA exists in the same resource block. Therefore SINR becomes the dominant QoS factor in NOMA system. Assume the received average SINRs at the BS from different users are equal, i.e. equal average SINR scheme, the average SINR becomes

$$
E\left[\gamma^{x_{1}}\right]=E\left[\gamma^{x_{2}}\right] .
$$

From Eq. (4), the second term can be written by

$$
E\left[\gamma^{x_{2}}\right]=\rho_{2}
$$

By replacing Eq. (2) into Eq. (12),

$$
E\left[\gamma^{x_{1}}\right]=E\left[\frac{\rho_{1}\left|h_{1}\right|^{2}}{\rho_{2}\left|h_{2}\right|^{2}+1}\right] .
$$

Define $x=\rho_{1}\left|h_{1}\right|^{2}$ and $y=\rho_{2}\left|h_{2}\right|^{2}+1$, where $x \geq 0$ and $y \geq 1$. Then Eq. (14) can be rearranged by

$$
\begin{aligned}
E\left[\gamma^{x_{1}}\right] & =E\left[\frac{x}{y}\right]=\int_{y=1}^{\infty} \int_{x=0}^{\infty} \frac{x}{y} f_{X, Y}(x, y) d x d y \\
& =\int_{y=1}^{\infty} \int_{x=0}^{\infty} \frac{x}{y} f(x) f(y) d x d y
\end{aligned}
$$

where $f_{X, Y}(x, y)$ is the joint pdf of $\mathrm{X}$ and $\mathrm{Y}$. The last equality holds from independence between $X$ and $Y$. The random variables of $\mathrm{X}$ and $\mathrm{Y}$ have exponential pdf, Eq. (15) becomes

$$
\begin{aligned}
& E\left[\gamma^{x_{1}}\right]= \\
& \int_{y=1}^{\infty} \int_{x=0}^{\infty} \frac{x}{y}\left(\frac{1}{\rho_{1}} e^{-\frac{x}{\rho_{1}}}\right)\left(\frac{1}{\rho_{2}} e^{-\frac{(y-1)}{\rho_{2}}}\right) d x d y \\
& =\frac{1}{\rho_{1} \rho_{2}} \int_{y=1}^{\infty} \emptyset \frac{1}{y} e^{-\frac{(y-1)}{\rho_{2}}} d y
\end{aligned}
$$

where

$$
\varnothing=\int_{x=0}^{\infty} x e^{-x / \rho_{1}} d x
$$

With the help of the following identity [15, Eq. (8.336.2)]

$$
\int_{0}^{\infty} x^{m} \exp \left(-\beta x^{n}\right) d x=\frac{\Gamma(\gamma)}{n \beta^{\gamma}}, \quad \gamma=\frac{m+1}{n},
$$

Eq. (17) can be simplified by

$$
\varnothing=\rho_{1}^{2}
$$


Replace Eq. (19) into Eq. (16), we have

$$
E\left[\gamma^{x_{1}}\right]=\frac{\rho_{1}}{\rho_{2}} \int_{0}^{\infty} \frac{1}{z+1} e^{-z / \rho_{2}} d z
$$

Also, with the help of the following identity [15, Eq. (3.352.4)]

$$
\int_{0}^{\infty} \frac{e^{-\mu x}}{x+\beta} d x=-e^{\beta \mu} E_{i}(-\beta \mu),
$$

where $E_{i}(\cdot)$ is the exponential integral function [15, Eq. (3.351.5)],

$$
E_{i}(-\mu)=-\int_{1}^{\infty} \frac{e^{-\mu x}}{x} d x
$$

Eq. (20) can be rewritten by

$$
E\left[\gamma^{x_{1}}\right]=\frac{\rho_{1}}{\rho_{2}}\left\{-e^{1 / \rho_{2}} E_{i}\left(-\frac{1}{\rho_{2}}\right)\right\}
$$

By replacing Eq. (12) and Eq. (13) into Eq. (23), we can obtain

$$
\rho_{1}=-\frac{\rho_{2}^{2}}{e^{1 / \rho_{2} E_{i}\left(-1 / \rho_{2}\right)}} .
$$

Furthermore, $E_{i}(x)$ can be rewritten by series term [15, Eq. (8.214.1)]

$$
E_{i}(x)=C+\ln (-x)+\sum_{k=1}^{\infty} \frac{x^{k}}{k \cdot k !}, x<0
$$

where $C$ is Euler's constant of 0.5772. Substitute Eq. (25) for Eq. (24), we can obtain the average SNR $\rho_{1}$ at the BS from UE1 as

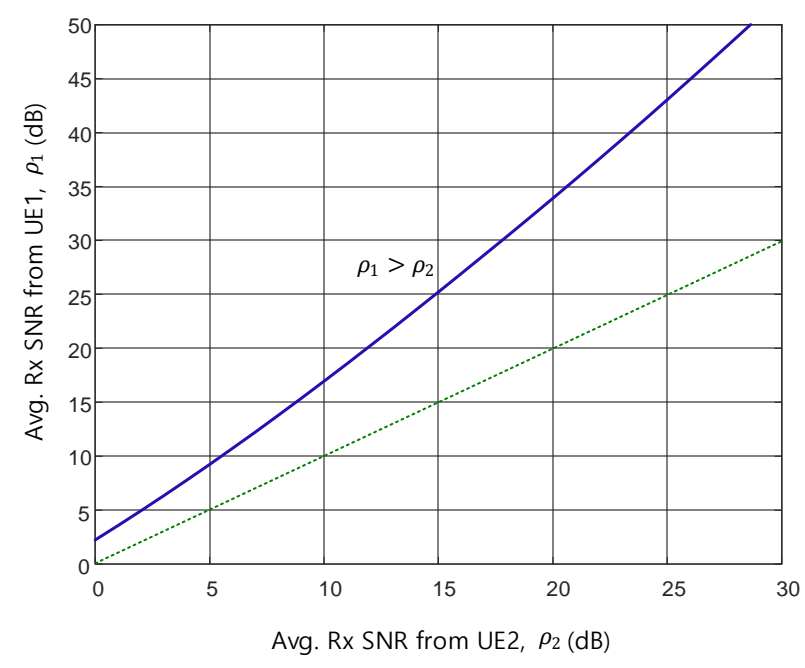

Figure. $2 \rho_{1}$ vs. $\rho_{2}$

$$
\rho_{1}=-\frac{\rho_{2}^{2}}{e^{1 / \rho_{2}}\left\{C-\ln \rho_{2}+\sum_{k=1}^{\infty}\left(-\frac{1}{\rho_{2}}\right)^{k} \frac{1}{k \cdot k !}\right\}} .
$$

Put Eq. (26) into Eqs. (6) and (11), the outage probabilities of UE1 and UE2 can be obtained, respectively.

Fig. 2 shows the relationship between the average SNR $\rho_{1}$ and $\rho_{2}$ in Eq. (26) with solid line under the condition of the equal SINR at the BS. It is noticed that $\rho_{1}$ is always greater than $\rho_{2}$. The slotted line indicates that $\rho_{1}$ equals $\rho_{2}$ for comparison. Since the noise power of $\rho_{1}$ and $\rho_{2}$ are equal, the received average signal strength from UE1 is greater than that of the UE2. For this reason, the decoding order in Eq. (4) is reasonable: decode the information of UE1 first and decode UE2 after SIC.

\subsubsection{Conventional power back-off scheme}

As we mentioned in the introduction, a far user in a cell transmits less power than a near user in conventional power back-off scheme. The received average SNR at the BS, $E\left[\gamma_{b}^{x_{2}}\right]=\rho_{2 \_} b$, in power back-off scheme is given by [12-13]

$$
\rho_{2 \_b}(d B)=\rho_{1}-\delta
$$

where $\delta(\mathrm{dB})$ denotes back-off power. And $\rho_{1}$ and $\rho_{2_{-} b}$ are in $\mathrm{dB}$. Therefore, the outage probabilities of UE1 and UE2 in power back-off scheme can be obtained by replacing $\rho_{2}$ in Eq. (6) and Eq. (11) with $\rho_{2 \_b}$ in Eq. (27), respectively.

\subsection{Ergodic capacity}

In NOMA system, the ergodic capacity can be written as

$$
\mathcal{R}=\mathrm{E}\left[\log _{2}(1+\gamma)\right]=\log _{2}(1+E[\gamma])
$$

where $\gamma$ is the received SINR. The capacity of UE1 and UE2 can be obtained by replacing $E[\gamma]$ with $E\left[\gamma^{x_{1}}\right]$ and $E\left[\gamma^{x_{2}}\right]$, respectively. As shown in Eq. (13), the average SINR of UE1 and UE2 are equal, hence, the ergodic capacities of near and far users are identical in the proposed scheme. While the capacities of UE1 and UE2 in power back-off scheme can be derived by substituting Eqs. (23) and (27) for $E[\gamma]$ in Eq. (28), respectively.

\section{Numerical examples}

This section describes the numerical examples of the derived outage probability and ergodic capacity of users. The analysis and Monte Carlo simulation 


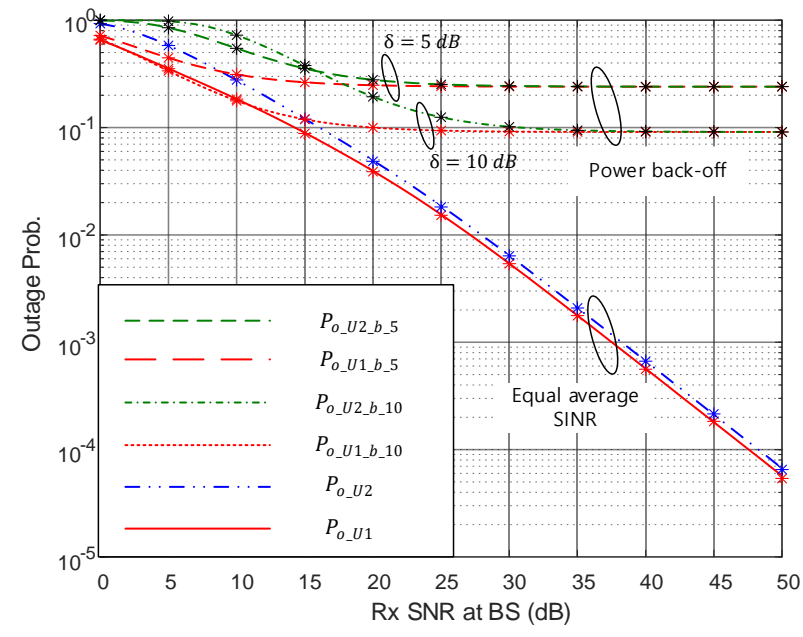

Figure. 3 Outage probability of UE1 and UE2 $\left(R_{1}=R_{2}=1[\mathrm{bps} / \mathrm{Hz}]\right)$

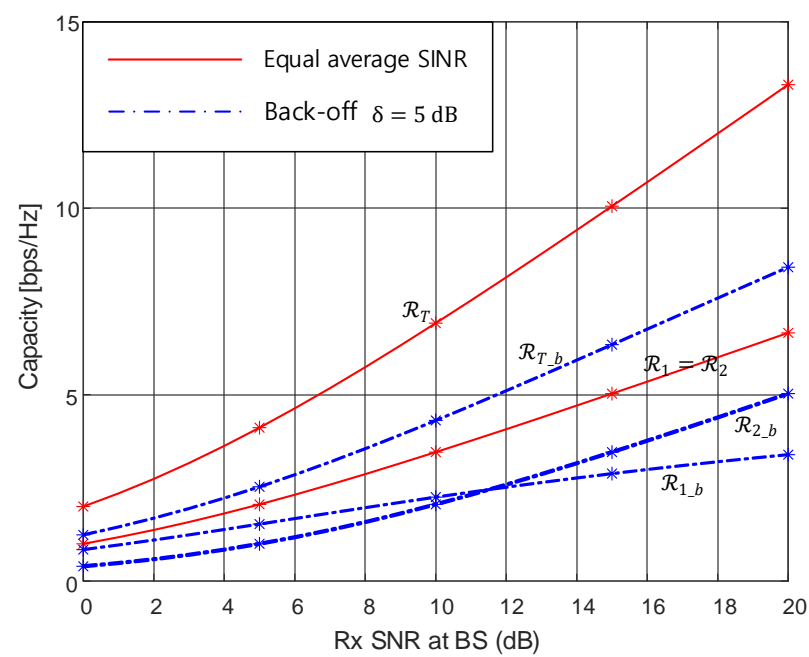

Figure. 4 Ergodic capacity

with $1 \times 10^{7}$ iterations are compared. The simulation results denote "*" in figures.

The outage probabilities of UE1 and UE2, $P_{O_{-} U 1}$ and $P_{O_{-} U 2}$, are shown in Fig. 3. The horizontal axis denotes the received average SNR at the BS from UE2. In this figure, it is noted that the outage probability of UE2 is slightly higher than the UE2. The reason is that the outage probability of UE2 is the sum of the outage probability of UE1 (the first term) and the second positive term in Eq. (7). Also the outage probability of UE2 is gradually approaching to that of UE1 with the increase of the SNR.

The outage probabilities of UE1 and UE2 with back-off power $\delta=5 \mathrm{~dB}(10 \mathrm{~dB}), P_{o_{-} U 1_{-} b_{5} 5}$ and $P_{O_{-} U 2_{-} b_{-} 5}\left(P_{O_{-} U 1 b_{-} b_{1} 10}\right.$ and $\left.P_{O_{-} U 2_{-} b_{-} 10}\right)$, in power back-off scheme are also shown in Fig. 3. It is noticed that the outage probability decreases as the back-off power increases. Also note that the error floor with the power back-off scheme. However, in the proposed scheme, the outage probabilities of UE1 and UE2 decrease as the increase of the received SNR without error floor. The simulation results are matched well with the analysis. It shows that the analytical expressions have been validated through simulation.

The ergodic capacity is shown in Fig. 4, where $\mathcal{R}_{1}$ and $\mathcal{R}_{2}$ denote the capacity [bps/Hz] of UE1 and UE2 of the proposed equal SINR scheme, respectively. Also $\mathcal{R}_{T}$ is the Sum capacity, $\mathcal{R}_{T}=\mathcal{R}_{1}+\mathcal{R}_{2}$. As we mentioned in section III, the capacities of UE1 and UE2 are identical to each other due to the equal SINR at the BS in Eq. (12).

Similarly, $\mathcal{R}_{1_{b} b}, \mathcal{R}_{2_{-} b}$, and $\mathcal{R}_{T_{-} b}$ denote the capacities of UE1, UE2, and sum capacity of the power back-off scheme with $\delta=5 \mathrm{~dB}$, respectively. When the received SNR bellows about $11.5 \mathrm{~dB}$, the capacity of UE1 $\left(\mathcal{R}_{1_{-} b}\right)$ is greater than UE2 $\left(\mathcal{R}_{2_{-} b}\right)$, and vice versa. The reason is that the higher received power from UE2 causes higher interference to UE1. Analytical results are conformed through comparison with simulations.

\section{Conclusions}

In this study, we propose an equal average SINR scheme which receives identical average SINR at the $\mathrm{BS}$, irrespective of the location of the users in uplink NOMA systems. The outage probability and the ergodic capacity are derived in closed-form expressions, and verified through simulation.

Numerical results show the outage probabilities of the proposed scheme do not exist error floor, while conventional power back-off scheme exist. It is noticed that the outage probabilities of near and far users decrease with the increase of the SNR at the BS. Also the outage probability of UE2 is gradually approaching to that of UE1 with SNR.

There are capacity unbalances between near and far users in power back-off scheme, however, that is identical in the proposed scheme. We can conclude that the outage probability between near and far users are very close and the ergodic capacity can be kept identical in the proposed scheme. Therefore our proposed scheme can be applied to maintain the performance balances between the users in uplink NOMA systems.

Further study will be focused on the performance and capacity improvement of uplink NOMA systems.

\section{Conflicts of Interest}

The authors declare no conflict of interest.

\section{References}

[1] Z. Ding, Y. Liu, J. Choi, Q. Sun, M. Elkashlan, C.-L. I, and V. Poor, "Application of Non- 
Orthogonal Multiple Access in LTE and 5G Networks", IEEE Communications Magazine, Vol. 55, No. 2, pp. 185-191, 2017.

[2] L. Dai, B. Wang, Y. Yuan, S. Han, C.-L. I, and Z. Wang, "Non-Orthogonal Multiple Access for 5G: Solutions, Challenges, Opportunities, and Future Research Trends", IEEE Communications Magazine, Vol. 53, No. 9, pp. 74-81, 2015.

[3] L. Dai, B. Wang, Z. Ding, Z. Wang, S. Chen, and L. Hanzo, "A Survey of Non-Orthogonal Multiple Access for 5G", IEEE Communications Survey and Tutorials, Vol. 20, No. 3, pp. 22942323, 2018.

[4] Y. Saito, Y. Kishiyama, A. Benjebbour, T. Nakamura, A. Li, and K. Higuchi, "NonOrthogonal Multiple Access (NOMA) for Cellular Future Radio Access", In: Proc. of Vehicular Technology Conf. (VTC Spring), Dresden, Germany, pp. 1-5, 2013.

[5] Y. Liu, Z. Ding, M. Elkashlan, and V. Poor, "Cooperative Non-Orthogonal Multiple Access with Simultaneous Wireless Information and Power Transfer", IEEE Journal on Selected Areas in Communications, Vol. 34, No. 4, pp. 938-953, 2016.

[6] C. Zhong and Z. Zhang, "Non-Orthogonal Multiple Access with Cooperative Full-Duplex Relaying", IEEE Communications Letters, Vol. 20, No. 12, pp. 2478-2481, 2016.

[7] N. -S. Kim, "Performance of User Relaying Cooperative NOMA System Underlay Cognitive Radio Networks with Partial CSI", International Journal of Intelligent Engineering and Systems, Vol. 12, No. 2, pp. 287-296, 2019.

[8] N. -S. Kim, "Outage Performance of CoMP NOMA Networks with Selective Cell and Transmit Diversity", International Journal of Intelligent Engineering and Systems, Vol. 13, No. 4, pp. 196-203, 2020.

[9] F. Wei, T. Zhou, T. Xu, and H. Hu, "BER Analysis for Uplink NOMA in Asymmetric Channels", IEEE Communications Letters, (Early Access), DOI: 10.1109/LCOMM.2020.3003274, 2020.

[10] Y. Sun, Z. Ding, X. Dai, and O. A. Dobre, "On the Performance of Network NOMA in Uplink CoMP Systems: A Stochastic Geometry Approach", IEEE Transactions on Communications, Vol. 67, No. 7, pp. 5084-5098, 2019.

[11] S. A.-Razeq, S. Zhou, R. Bansal, and M. Zhao, "Uplink NOMA Transmissions in a Cooperative Relay Network Based on Statistical Channel
State Information", IET Communications, Vol. 13, No. 4, pp. 371-378, 2019.

[12] N. Zhang, Jing. Wang, G. Kang, and Yang Liu, "Uplink Nonorthogonal Multiple Access in 5G System", IEEE Communications Letters, Vol. 20, No. 3, pp. 458-461, 2016.

[13] Y. Gao, B. Xia, K. Xiao, Z. Chen, X. Li, and S. Zhang, "Theoretical Analysis of the Dynamic Decode Ordering SIC Receiver for Uplink NOMA Systems", IEEE Communications Letters, Vol. 21, No. 10, pp. 2246-2249, 2017.

[14] H. Liu, T. A. Tsiftsis, K. J. Kim, K. S. Kwak, and H. V. Poor, "Rate Splitting for Asynchronous Uplink NOMA Systems with Cyclic Prefix Single Carrier", In: Proc. of International Conf. on Communications Workshops (ICC Workshops), DOI: 10.1109/ICCW.2019.8756819, Shanghai, China, 2019.

[15] I. S. Gradshteyn and I. M. Ryzbik, Table of Integrals, Series, and Products, $6^{\text {th }}$ Ed., Academic Press, San Diego, CA 2002. 